\title{
Bark tissue transcriptome analyses of inverted Populus yunnanensis cuttings reveal the crucial role of plant hormones in response to inversion
}

\author{
An-Pei Zhou ${ }^{1,2}$, Pei-Hua Gan ${ }^{1,2}$, Dan Zong ${ }^{1,2}$, Xuan Fei ${ }^{1,2}$, Yuan-Yuan Zhong ${ }^{1,2}$, Si-Qi Li ${ }^{1,2}$, Jin-De Yu ${ }^{1}$, Cheng- \\ Zhong He ${ }^{\text {Corresp. 1, 2, } 3}$ \\ ${ }^{1}$ Key Laboratory for Forest Genetic and Tree Improvement and Propagation in Universities of Yunnan Province, Southwest Forestry University, Kunming, \\ China \\ ${ }^{2}$ Key Laboratory of Biodiversity Conservation in Southwest China, State Forestry Administration, Southwest Forestry University, Kunming, China \\ 3 Key Laboratory for Forest Resources Conservation and Utilization in the Southwest Mountains of China, Ministry of Education, Southwest Forestry \\ University, Kunming, China \\ Corresponding Author: Cheng-Zhong He \\ Email address: hecz@swfu.edu.cn
}

Inverted cuttings of Populus yunnanensis exhibit an interesting growth response to inversion. This response is characterized by enlargement of the stem above the shoot site, while the upright stem shows obvious outward growth below the shoot site. In this study, we examined transcriptome changes in bark tissue at four positions on upright and inverted cuttings of $P$. yunnanensis: position $B$, the upper portion of the stem; position $C$, the lower portion of the stem; position $D$, the bottom of new growth; and position $E$, the top of new growth. The results revealed major transcriptomic changes in the stem, especially at position $B$, but little alteration was observed in the bark tissue of the new shoot. The differentially expressed genes (DEGs) were mainly assigned to four pathways: plant hormone signal transduction, plant-pathogen interaction, mitogen-activated protein kinase (MAPK) signaling pathway-plant, and adenosine triphosphate-binding cassette $(A B C)$ transporters. Most of these DEGs were involved in at least two pathways. The levels of many hormones, such as auxin (IAA), cytokinin (CTK), gibberellins (GAs), ethylene (ET), and brassinosteroids (BRs), underwent large changes in the inverted cuttings. A coexpression network showed that the top 20 hub unigenes at position $B$ in the upright and inverted cutting groups were associated mainly with the BR and ET signaling pathways, respectively. Furthermore, brassinosteroid insensitive 1-associated receptor kinase 1 (BAK1) in the BR pathway and both ethylene response (ETR) and constitutive triple response 1 (CTR1) in the ET pathway were important hubs that interfaced with multiple pathways. 
1 Bark tissue transcriptome analyses of inverted

2 Populus yunnanensis cuttings reveal the crucial role 3 of plant hormones in response to inversion

5 An-Pei Zhou ${ }^{1,2}$, Pei-Hua Gan ${ }^{1,2}$, Dan Zong ${ }^{1,2}$, Xuan Fei ${ }^{1,2}$, Yuan-Yuan Zhong ${ }^{1,2}$, Si-Qi Li1 ${ }^{1,2}$, Jin-

6 De $\mathrm{Yu}^{1}$, Cheng-Zhong $\mathrm{He}^{1,2,3}$

$7 \quad{ }^{1}$ Key Laboratory for Forest Genetic and Tree Improvement and Propagation in Universities of

8 Yunnan Province, Southwest Forestry University, Kunming 650224, China

$9 \quad{ }^{2}$ Key Laboratory of Biodiversity Conservation in Southwest China, State Forestry

10 Administration, Southwest Forestry University, Kunming 650224, China

$11{ }^{3}$ Key Laboratory for Forest Resources Conservation and Utilization in the Southwest Mountains

12 of China, Ministry of Education, Southwest Forestry University, Kunming 650224, China

14 Corresponding Author:

15 Cheng-Zhong $\mathrm{He}^{1,2,3}$

16 No.300 Bailong Road, Kunming City, Yunnan Province, 650224, China

17 Email address: hecz@swfu.edu.cn 


\section{Abstract}

20 Inverted cuttings of Populus yunnanensis exhibit an interesting growth response to inversion.

21 This response is characterized by enlargement of the stem above the shoot site, while the upright

22 stem shows obvious outward growth below the shoot site. In this study, we examined

23 transcriptome changes in bark tissue at four positions on upright and inverted cuttings of $P$.

24 yunnanensis: position $\mathrm{B}$, the upper portion of the stem; position $\mathrm{C}$, the lower portion of the stem;

25 position $\mathrm{D}$, the bottom of new growth; and position $\mathrm{E}$, the top of new growth. The results

26 revealed major transcriptomic changes in the stem, especially at position $\mathrm{B}$, but little alteration

27 was observed in the bark tissue of the new shoot. The differentially expressed genes (DEGs)

28 were mainly assigned to four pathways: plant hormone signal transduction, plant-pathogen

29 interaction, mitogen-activated protein kinase (MAPK) signaling pathway-plant, and adenosine

30 triphosphate-binding cassette (ABC) transporters. Most of these DEGs were involved in at least

31 two pathways. The levels of many hormones, such as auxin (IAA), cytokinin (CTK), gibberellins

32 (GAs), ethylene (ET), and brassinosteroids (BRs), underwent large changes in the inverted

33 cuttings. A coexpression network showed that the top 20 hub unigenes at position B in the

34 upright and inverted cutting groups were associated mainly with the BR and ET signaling 35 pathways, respectively. Furthermore, brassinosteroid insensitive 1-associated receptor kinase 1

36 (BAK1) in the BR pathway and both ethylene response (ETR) and constitutive triple response 1

37 (CTR1) in the ET pathway were important hubs that interfaced with multiple pathways. 
39

40

41

42

43

44

45

46

47

48

49

50

51

52

53

54

55

56

57

58

59

60

61

62

63

64

65

66

67

68

69

70

71

72

73

74

75

76

77

78

\section{Introduction}

Plant polarity, an essential feature of differentiation along an axis of symmetry, designates the specific orientation of activity in space (Belanger \& Quatrano, 2000; Qi et al., 2017). Studies in algae have suggested that an initial asymmetric division of the zygote plays a key role in the determination of different cell fates in early embryonic cells (Brownlee et al., 2001; Hable \& Hart, 2010). The polar organization of molecules and cellular structures could significantly cause developmental changes in the morphogenesis, growth and function of cells and could allow specialized cell types to perform individual tasks (Belanger \& Quatrano, 2000; Brownlee et al., 2001; Euchen et al., 2012). The flow of determination from the cellular to the tissue level predetermines the formation of various tissues with high polarity, such as root hairs and pollen tubes (Grebe, 2004).

Shoot-root polarity divides the plant body into two parts, the root at the base and the shoot at the top, and their apices point in opposite directions (Nick \& Furuya, 1992). Baskin et al. (2010) proposed an apex-based terminology for plant polarity, including the new terms "shootward" and "rootward", meaning specifically toward the shoot apex and toward the root apex, respectively. In higher plants, water and mineral elements are absorbed mainly by root hairs and are transported shootward into the aboveground parts, while organic nutrients are mainly photosynthesized in the leaves and transported rootward into the underground parts. This arrangement ensures the growth and development of plants. However, we found that certain inverted trees could still grow, in contrast to the typical case of woody plant polarity.

Yunnan white poplar (Populus yunnanensis), an important forest tree species from the Tacamahaca section of the Populus genus in the Salicaceae family, is a native dioecious species widely distributed in southwestern China (Chen et al., 2010; Jiang et al., 2012). Owing to their rapid growth, strong adaptability, easy asexual propagation, cold resistance, $P$. yunnanensis trees have been planted for the greening of cities and roads, and play an important role in forestry production, afforestation and environmental conservation (Jiang et al., 2013; Li et al., 2014; Ren et al., 2018). By chance, we found that inverted P. yunnanensis cuttings could form complete plants. The roots grew in the shootward direction, and shoots grew in the rootward direction; this development was linked to the physical orientation and not to the original polarity. The new shoots of inverted cuttings displayed less vigorous growth than did those of upright cuttings (Zhou et al., 2018). During the next year, obvious enlargement occurred above the shoot site of the inverted cuttings, while the upright cuttings exhibited outward growth below the shoot site (Fig. 1). This growth against plant polarity is of interest.

\section{Location of Fig. 1}

Previous cases of shoot-root polarity were reported mainly during the 20th century (Bloch, 1943; Bünning, 1952; Sachs, 1969; Weisenseel, 1979; Nick \& Furuya, 1992). Based on the morphogenesis of inverted plants, researchers have proposed either the hypothesis that polarity is induced de novo or the opposing view that polarity is stable. Vöhting's experiment (Nick \& Furuya, 1992) with inverted dandelion roots showed that adventitious shoots formed at the root pole when the shoot pole had been sealed with resin. However, a segment cut from an inverted 
79

80

81

82

83

84

85

86

87

88

89

90

91

92

93

94

95

96

97

98

99

100

101

102

103

104

105

106

107

108

109

110

111

112

113

114

115

116

117

plant without sealing the poles could grow shoots at the original shoot pole, which suggested an unaltered original polarity.

Inverted cuttings are considered excellent materials for understanding plant polarity, which is involved mainly in morphological reconstruction and shoot-root polarity. To date, relevant research has focused on how plant polarity is induced and fixed and how it orients cell division (Souter \& Lindsey, 2000; Friml et al., 2006; Medvedev, 2012; Bringmann \& Bergmann, 2017; Strzyz, 2017; Bornens, 2018). Although clear different from that of upright plant, the growth and development of inverted plants has largely been ignored. The different characteristics of the two direction types are the consequence of original shoot-root polarity changes, and some factors involved in polar responses play important roles in this process. In this study, inverted $P$. yunnanensis cuttings were characterized as having dwarf-type new shoots and enlarged stems above the shoot site. We performed transcriptome profiling of bark tissues in both upright and inverted cuttings and examined the main changes in the stems and new shoots in response to inversion. These findings could reveal the most important factors impacting the growth of inverted plants and could help to understand their action in response to plant polarity changes.

\section{Materials and Methods}

Plant materials

Three one-year-old P. yunnanensis clones from the Haikou Forest Farm of Kunming (China) were selected as three biological replicates, and their main stems were used to generate cuttings of similar length and diameter. We cultivated the cuttings at Southwest Forestry University (Kunming, China) during early spring (March) in two orientations: upright and inverted. With the exception of the middle bud on each cutting, all the buds were removed. During early August (the vegetative growth stage), the shoot branch sprouting from this bud and its cutting body were collected, and we sampled bark tissues at the following four positions (Fig. S1) to reveal any changes induced by inversion: positions $\mathrm{B}$ and $\mathrm{C}$, which were above and below the shoot site, respectively; position $\mathrm{D}$, which was at the bottom of the new growth; and position $\mathrm{E}$, which was at the top. Four individuals with the same clonal origin, position and direction type were pooled to generate one mixed bark sample. A total of twenty-four bark samples from the four positions (three replicates each) were used to compare the transcriptome profiles of the two orientation treatments. The sample groups from the four positions (B, C, D, E) of the upright (U) and inverted (I) cuttings were named BU, CU, DU, EU, BI, CI, DI, and EI, and the three replicates within each group were numbered with 1 (e.g., BU1), 2 (e.g., BU2), and 3 (e.g., BU3).

\section{RNA isolation and sequencing library preparation}

Total RNA was isolated from each sample using an RNAprep Pure Plant Kit (Tiangen Biotech, Beijing, China). The RNA concentration and purity were subsequently assessed with a NanoDrop spectrophotometer (NanoDrop 1000, Thermo Scientific, Wilmington, Delaware, USA) and a Qubit fluorometer (Invitrogen, Carlsbad, California, USA), and the RNA integrity

Peer] reviewing PDF | (2019:04:36443:2:1:NEW 9 Aug 2019) 
118 was checked on an Agilent 2000 system (Agilent Technologies, Palo Alto, California, USA). 119 Each sample was used for cDNA library construction with a VAHTS Stranded mRNA-seq 120 Library Prep Kit for Illumina (NR602, Vazyme, Nanjing, Jiangsu, China). In this step, the 121 mRNA was purified from the total extracted RNA via oligo d(T) beads and was fragmented at 85 $122{ }^{\circ} \mathrm{C}$ for $6 \mathrm{~min}$. Random hexamers were used for first-strand cDNA synthesis, and second-strand 123 cDNA synthesis and end repair were performed via Second Strand/End Repair Enzyme Mix.

124

125

126

127

128

129

130

131

132

133

134

135

136

137

138

139

140

141

142

143

144

145

146

147

148

149

150

151

152

153

154

155

156 After purification via DNA Clean Beads, the double-stranded cDNA was processed with dA tailing and adapter ligation. The PCR amplification reaction was used to enrich the library. After quality control and quantification via an Agilent 2100 Bioanalyzer and a Qubit fluorometer, the qualified libraries were sequenced on an Illumina HiSeq X Ten device at $2 \times 150 \mathrm{bp}$, and each library produced approximately 6 gigabases $(\mathrm{Gb})$ of paired-end raw reads. The transcriptome data in this study were submitted to the short read archive (SRA) database under the accession number PRJNA506110.

\section{De novo assembly and functional annotation}

The raw Illumina reads were quality-trimmed and filtered to remove adapter sequences, reads containing poly-N sequences $(>5 \%)$, and low-quality reads $(\mathrm{Q}<20)$ by in-house Perl scripts. The clean reads were used for de novo assembly with Trinity (Grabherr et al., 2011). In brief, the short reads were first combined to generate linear contigs with a certain length of overlap based on $\mathrm{K}$-mers values $(\mathrm{K}=25)$. The contigs, which were comprised of alternative splicings and paralogous genes, were clustered to generate unigenes using the De Bruijn graph algorithm. The sequences were clustered to eliminate the redundant contigs using TGICL (Pertea et al., 2003). The generated unigenes were annotated using BLAST alignment tools (Altschul et al., 1990). A total of ten nucleotide and protein databases, namely, the nonredundant nucleotide sequences (NT), non-redundant protein sequences (NR), Swiss-Prot, clusters of orthologous groups of proteins (COG), gene ontology (GO), Kyoto encyclopedia of genes and genomes (KEGG), plant transcription factor database (PlantTFdb), plant resistance gene database (PRGdb), InterPro, and search tool for the retrieval of interacting genes/proteins database (STRINGdb), were used to determine the unigene functions. The parameters of these programs are listed in Table $\mathrm{S} 1$.

\section{Detection of DEGs}

We calculated the fragments per kilobase of transcript per million mapped reads (FPKM) to assess the expression levels of every sample using RSEM software ( $L i \&$ Dewey, 2011). On the basis of read count, the differentially expressed genes (DEGs) between pairs of sample groups were detected using the DESeq package (Anders \& Huber, 2010), and we chose those with a false discovery rate $(F D R) \leq 0.05$ and a fold change $(F C) \geq 1$. These DEGs were further divided into two patterns: upregulation and downregulation. To assess the functions of the DEGs, a hypergeometric distribution test was applied to identify significantly enriched GO terms and 
157 KEGG pathways with TBtools (Chen et al., 2018), and their visualization was carried out with

158

159

160

161

162

163

164

165

166

167

168

169

170

171

172

173

174

175

176

177

178

179

180

181

182

183

184

185

186

187

188

189

190

191

192

193

194

195

the ggplot2 package (Wickham, 2016).

\section{Hub unigene search}

When we compared the transcriptome differences between the control and treated samples, some hub unigenes were ignored by the DEG decision protocol. We thus used a weighted gene co-expression network analysis (WGCNA) (Langfelder \& Horvath, 2008) to explore the coexpression of unigenes in highly enriched KEGG pathways, in which there were large numbers of DEGs. First, the soft power was determined via a soft-threshold approach, and the unigenes were clustered into coexpression modules. Then, the sample group information was imported, and the module-trait relationships were examined to search for key modules. Finally, the unigene interactions in these modules were visualized using Cytoscape software (Shannon et al., 2003; Cline et al., 2007), and a gene coexpression network based on hit number was constructed. Considering the expression information, we marked these unigenes and confirmed the top 20 hub unigenes per group.

\section{Analysis of RT-qPCR}

To validate the RNA sequencing (RNA-seq) results, we chose ten hub unigenes from the WGCNA and quantified them using real-time quantitative PCR (RT-qPCR). The RNA extracted for library preparation and transcriptome sequencing was used as a template for cDNA synthesis, and reverse transcription was performed using a FastQuant RT Kit (Tiangen Biotech, Beijing, China). EVA Green (Fast Super EvaGreen qPCR Master Mix, US Everbright Inc., USA) chemistry and a real-time PCR system (Rotor-Gene Q, Qiagen, Germany) were used to validate the hub unigene expression. We used the endogenous control gene PD-EI (Pyruvate Dehydrogenase E1) to quantify the relative mRNA levels (Carraro et al., 2012; Yun et al., 2019). The primers of the hub unigenes were designed using Primer Premier 5 (Lalitha, 2000) and synthesized by Sangon Biotech Co., Ltd. (Shanghai, China). Information on all the primers used is listed in Table S2. Two-step amplification was carried out as follows: $95{ }^{\circ} \mathrm{C}$ for $2 \mathrm{~min}$, followed by 40 cycles of $95{ }^{\circ} \mathrm{C}$ for $10 \mathrm{~s}$ and $60{ }^{\circ} \mathrm{C}$ for $30 \mathrm{~s}$. The relative expression level was calculated by the $2^{-\Delta \Delta \mathrm{Ct}}$ method (Livak \& Schmittgen, 2001). This experiment was repeated for three biological replicates, each involving three technical repeats.

\section{Results}

\section{Transcriptome sequencing, de novo assembly and functional annotation}

The RNA-seq libraries generated an average of 45,829,779 raw reads $(40,920,000$ $50,911,246)$. After quality control, an average of $44,153,404$ clean reads were obtained from the 24 samples $(39,078,392 \sim 49,171,196)$. With Q20 > 96.65\% and Q30 > 91.64\%, more than 105 thousand unigenes per sample were assembled, and their N50 values were greater than 1436 (Table S3). These results indicated that the RNA-seq data were of high quality. 
196

197

198

199

200

201

202

203

204

205

206

207

208

209

210

211

212

213

214

215

216

217

218

219

220

221

222

223

224

225

226

227

228

229

230

231

232

233

234

Among the 275,575 total unigenes, 208,387 (75.62\%) were annotated to ten databases (Fig. 2), and $71 \%$ were aligned to Populus trichocarpa in the NR database (Fig. S2). This annotation proportion was relatively low, suggesting a number of low-reliability unigenes. We therefore filtered the unigenes from each sample according to a criterion of FPKM $<1$. As a result, there were a total of 204,840 unigenes, of which 174,264 (85.07\%) corresponded to functional annotations (Fig. 2). After filtering, these unigenes were used as a basis for subsequent analyses.

Location of Fig. 2

\section{Detection of DEGs between upright and inverted samples}

We compared the pairwise unigene expression changes in P. yunnanensis samples from the same or different positions in different orientations (Fig. 3) and found obvious pattern differences. Total of 9,590 and 6,785 DEGs were found in the pairs BU vs BI and BU vs CI, respectively, which were the largest numbers among all the pairs. At position $\mathrm{C}$ in the upright $P$. yunnanensis samples, 339 and 754 DEGs were identified in the pairs CU vs CI and CU vs BI, respectively. Few DEGs were found at the two positions in the new growth (D and E): 74 in DU vs DI and 18 in EU vs EI. These results suggested that the transcriptome changes induced by inversion were major in the older cutting regions but that there was little influence on the new growth.

Location of Fig. 3

The DEGs in four pairs (BU vs BI, BU vs CI, CU vs CI, CU vs BI) of cuttings were important for understanding the transcriptome changes (Table S4). The GO database was used to determine their enrichment, as shown in Fig. 4. Of the three main categories, biological process had the most GO terms in all four pairs (BU vs BI: 24; BU vs CI: 21; CU vs CI: 17; CU vs BI: 17), followed by cellular component (BU vs BI: 16; BU vs CI: 14; CU vs CI: 11; CU vs BI: 15). In the biological process category, metabolic process and cellular process were the most abundant terms. In the cellular component category, the most enriched terms were cell, cell part, membrane, organelle, and membrane part. In the molecular function category, many DEGs were related to binding and catalytic activity.

\section{Location of Fig. 4}

The DEGs were also subjected to KEGG annotation (Table S4). As shown in the KEGG enrichment results (Fig. 5), plant hormone signal transduction and plant-pathogen interaction contained the most DEGs in the pairs BU vs BI and BU vs CI, and they were significantly enriched in these two pathways. The pairs CU vs CI and CU vs BI had the most DEGs with significant enrichment in plant hormone signal transduction. All four pairs showed dramatic differences in plant hormone signal transduction. Among the DEGs in CU vs CI, adenosine triphosphate-binding cassette $(\mathrm{ABC})$ transporters, which are members of a transport system superfamily, were significantly enriched. Notably, the number of DEGs in mitogen-activated protein kinase (MAPK) signaling pathway-plants was always high, indicating a clear effect of inversion on this pathway.

Location of Fig. 5 
235

236

237

238

239

240

241

242

243

244

245

246

247

248

249

250

251

252

253

254

255

256

257

258

259

260

261

262

263

264

265

266

267

268

269

270

271

272

A number of DEGs were associated with two or more pathways (Fig. 6). In the plant hormone signal transduction pathway, most DEGs in BU vs BI were related to auxin (also known as indole-3-acetic acid, IAA), cytokinin (CTK) and brassinosteroids (BRs) signaling, and the DEGs in CU vs BI were assigned mainly to ethylene (ET) signaling. All four of these hormones, as well as gibberellins (GAs), were associated with a large number of DEGs in BU vs CI. Most of these unigenes were upregulated in the pairs BU vs BI, BU vs CI and CU vs BI, and the three biological replicates exhibited high homogeneity of expression.

Location of Fig. 6

\section{Gene coexpression network construction}

We chose all the unigenes assigned to four KEGG pathways, including plant hormone signal transduction, plant-pathogen interaction, MAPK signaling pathway-plant, and ABC transporters, and grouped them into gene coexpression modules (Fig. 7). There was no clear outlier in the sample dendrogram. On the basis of an appropriate soft power of 10, the WGCNA divided these unigenes into 40 color modules. We examined these modules via the correlation between sample groups and gene modules and found that the hub modules were turquoise for $\mathrm{BU}$, light green for BI, orangered4 and dark turquoise for $\mathrm{CU}$, and pale turquoise for $\mathrm{CI}$.

\section{Location of Fig. 7}

The gene coexpression network including the DEG information revealed hub unigenes in each group (Fig. 8). The turquoise module was highly related to the BU group (0.904) and slightly related to the BI group (-0.060). Of the top 20 hub unigenes shown in Table 1,8 could encode brassinosteroid insensitive 1-associated receptor kinase 1 (BAK1), which is involved in BR signal transduction. Among the gene modules that were slightly related to the BU group, the light green module was most correlated with the BI group (0.756). Among the top 20 hub unigenes, a total of 16 were annotated with 4 compounds involved in the signal transduction of ET. These results indicated that some transcripts were inhibited and that some new functions were activated at position B when the cuttings were exposed to inverted conditions, and these changes approached significance. The correlation coefficients of the darkturquoise and orangered4 modules were high for the CU group but low for the CI group, and the correlation of the paleturquoise module was the opposite. A few genes in these three modules were differentially expressed.

Location of Fig. 8
Location of Table 1

\section{RT-qPCR verification}

The gene expression levels of ten hub unigenes (Table S5) obtained from the WGCNA results were validated via RT-qPCR (Fig. S3). Of these genes, six were downregulated, and four were upregulated. They all matched well with the RT-qPCR results, which corroborated the reliability of the RNA-seq results. 


\section{Discussion}

\section{BRs and ET are important hubs for changes in hormone signaling}

275 Plants coordinate their growth and development to adjust to the external environment 276 (Santner \& Estelle, 2009). This adjustment is a complex biological process in which hormones 277 play a role. Plant hormones, including IAA, CTK, GAs, abscisic acid (ABA), ET, BRs, jasmonic 278 acid (JA), and salicylic acid (SA), function in various aspects of growth, either singlehandedly or 279 interactively (Santner et al., 2009; Band et al., 2012; Pacifici et al., 2015). This complexity 280 arises from hormone biosynthesis, transport, and signaling pathways and from the diversity of

281

282

283

284

285

286

287

288

289

290

291

292

293

294

295

296

297

298

299

300

301

302

303

304

305

306

307

308

309

310

311

312 interactions between hormones. In the bark transcriptome analysis performed in this study, a number of DEGs were annotated to the plant hormone signal transduction pathway, including all the hormones in this map, which indicated a large influence of inversion on hormone signaling pathways. The coexpression network also showed that the top 20 hub unigenes were mainly part of two hormone signaling pathways: those involving BRs and ET.

BRs are major growth-promoting steroid hormones that modulate cell elongation and division (Mandava, 1988; Clouse \& Sasse, 1998; Belkhadir\& Jaillais, 2015; Vragovic et al., 2015). In addition to playing important roles in root growth and development, BRs actively function in stem elongation and vascular differentiation (Singh \& Savaldi-Goldstein, 2015; Ahanger et al., 2018). BR-insensitive mutants of Arabidopsis thaliana are characterized by multiple deficiencies in developmental pathways, and these mutants exhibit severe dwarfing, dark green color, thickened leaves, and reduced apical dominance (Clouse et al., 1996; Bishop, 2003). BRs have also emerged as crucial regulators of the growth-immunity trade-off (Krishna, 2003; De Bruyne, et al., 2014; Lozano-Duran \& Zipfel, 2015). Serving as a key mediator of environmental stress factors, ET is a gaseous hormone involved in many developmental processes and responses to biotic and abiotic stresses in plants (Corbineau et al., 2014; Müller \& Munné-Bosch, 2015; Thao et al., 2015; Broekgaarden et al., 2015; Berrabah et al., 2018). In this study, these two hormones were associated with hub unigenes detected in the bark tissues of the inverted cuttings of $P$. yunnanensis. The top 20 hub unigenes in the BU and BI groups were involved mainly in the BR and ET signaling pathways, respectively. Furthermore, among the constituents of the BR signaling pathway, only BAK1 was identified, and this protein could be encoded by 8 of the top 20 hub unigenes. In the BI group, 16 of the top 20 hub unigenes were related to ET signaling. The ethylene response (ETR) protein was associated with the most unigenes (8). These results indicated that the main changes induced by inversion occurred in the BR and ET signaling pathways.

Moreover, the signaling pathways of other hormones in bark tissues also strongly impacted the growth and development of the inverted cuttings of $P$. yunnanensis. Multiple hormones are at play, and the cooperation and crosstalk between their signaling pathways are complex (Depuydt \& Hardtke, 2011), as reflected by the coexpression network in this study. Because of the typical triple response, ET is related to radial swelling of the stem, which could result in obvious enlargement above the shoot site of inverted cuttings. Various studies (Muday et al., 2012) have suggested that, by altering the signaling, synthesis and transport of IAA, ET affects many aspects 
313 of IAA-dependent seedling growth. Among all the hormones, IAA, which was enriched for most 314 DEGs, is a peculiar plant hormone because of its own polar transport route and functions in the 315 proliferation and elongation of cells (Santner A and Estelle, 2009; Tian et al., 2018), which could 316 contribute to the enlargement of the stem of inverted cuttings. Several researchers have 317 hypothesized that the polarity in inverted flowering plants cannot be reversed and that cuttings 318 are resistant to changes in the polar direction of IAA transport (Sachs, 1969; Nick \& Furuya, 319 1992; Friml et al., 2006). Despite these hypotheses, new downward flow seems to occur in 320 addition to the original upward polarity (Went, 1941). The clear increase in outward growth 321 below the shoot site of inverted cuttings (Fig. 1) supported the hypotheses concerning new polar

323

324

325

326

327

328

329

330

331

332

333

334

335

336

337

338

339

340

341

342

343

344

345

346

347

348

349

350

351 IAA. BRs play an important role in vascular differentiation (Fukuda, 2004; Caño-Delgado et al., 2004), which may induce the establishment of new cambium tissue in inverted plant cuttings to transport new IAA in the opposite direction in addition to old cambium that retains its IAA polarity.

\section{Hub unigenes also function in MAPK signaling and plant immunity}

Cutting inversion notably affected the MAPK signaling pathway and plant-pathogen interactions. A number of constituents in these two pathways were associated with the DEGs. For clear identification, we focused on the top 20 hub unigenes obtained from the WGCNA and coexpression network. In addition to their important roles in plant hormone signal transduction, many of these unigenes also actively function in MAPK signaling (all 20 unigenes) and plant immunity (10 unigenes), which indicates that close links among these three pathways and an important hub consisting of a few genes annotated to these pathways exist.

Some genes involved in hormone signal transduction such as BAK1 can function in the MAPK pathway and in plant-pathogen interactions (Li et al., 2002; Chinchilla et al., 2007). BAK1, which is also known as BAK1/SERK3, is the third member of the small somatic embryogenesis receptor kinase (SERK) family of Arabidopsis; it is a receptor-like kinase implicated in plant immunity and MAPK signaling (Nam \& Li, 2002; Heese et al., 2007). Recognition of microbe/pathogen -associated molecular patterns (MAMPs/PAMPs) are central to innate plant immunity (Yamada et al., 2016; Yasuda et al., 2017). In the MAPK pathway, the proteins BAK1 and Flagellin Sensing 2 (FLS2) form a receptor-like kinase complex located in the plasma membrane (Chinchilla et al., 2007). This complex recognizes a 22-amino acid peptide from flagellin (Flg22), a major structural protein of the eubacterial flagellum that acts as a PAMP in plants (Colcombet \& Hirt, 2008; Meng \& Zhang, 2013). In the plant-pathogen interaction pathway, BAK1 is recruited to FLS2 and elongation factor (EF)-TU receptor (EFR), which can recognize the bacterial MAMP flg22 epitope and EF-TU (elf18 epitope), respectively (Heese et al., 2007; Henry et al., 2013). When BAK1 is silenced, Nicotiana benthamiana exhibits attenuated resistance to bacterial and oomycete pathogens (Chaparro-Garcia et al., 2011). Therefore, BAK1 is an important hub that functions in various signaling pathways, and it was also identified as a hub in the altered bark transcription network induced by inversion in $P$. yunnanensis. 
352

353

354

355

356

357

358

359

360

361

362

363

364

365

366

367

368

369

370

371

372

373

374

375

376

377

378

379

380

381

382

383

384

385

386

387

388

389

390

391

Plant ET hormone signaling also involves MAPK cascades (Chang, 2003; Ouaked et al., 2003; Schweighofer \& Meskiene, 2008; Li et al., 2018), which are highly conserved signaling pathways across eukaryotes. Membrane-localized ETRs are similar to bacterial two-component histidine kinases and are encoded by ETR1, ETR2, ethylene resistant 1 (ERS1), ERS2, and EIN4 in Arabidopsis (Schweighofer \& Meskiene, 2008). Previous studies have revealed that ETRs are negative regulators and actively repress downstream components such as constitutive triple response 1 (CTR1), a MAPK kinase kinase (MAPKKK) that acts as a negative regulator in ET signaling (Hua \& Meyorowitz, 1998; Qu et al., 2007; Bakshi et al., 2015). In this study, ETR and CTR1 were encoded by 8 and 1 of the top 20 hub unigenes in the BI group, respectively, which indicated a strong effect on the receipt and transmission of ET after inversion in P. yunnanensis cuttings.

However, the genes related to plant immunity might not be directly related to polarity. A polar signal induced by inversion, which is similar to induction caused by external stress, might activate the immune response mechanism of plants. The genes related to hormone signaling first responded intensely to inversion, which also resulted in the activation of plant immunity as an incidental to hormone alteration because of sharing among common hub unigenes. This great attention to the "stress" could limit the growth and development of inverted plants. Despite a lack of actual chemical detection, it is believed that hormones play a crucial role in responding to inversion, but additional experiments are needed to verify the involvement of hormones in polarity change.

\section{Conclusions}

Cuttings of $P$. yunnanensis present an interesting growth response to inversion, characterized by enlarged stems and dwarf-type new shoots. Our study focused on transcriptome changes in bark tissue induced by cutting inversion. The results revealed the major transcriptome changes induced by inversion in the older cutting regions, but there was little influence on the new growth. Moreover, plant hormones played a crucial role in the inverted cuttings. The levels of many hormones, including IAA, CTK, GAs, ET, and BRs, underwent large changes in the inverted cuttings. Furthermore, BAK1 in the BR pathway and ETR and CTR1 in the ET pathway were important hubs that interacted with multiple pathways, such as MAPK signaling and plant immunity.

\section{Acknowledgments}

We thank Prof. Aizhong Liu, Kunming Institute of Botany, for his help with the experimental design.

\section{Supplemental Materials}

Table S1: Parameters of the programs used in the de novo assembly and functional annotation.

Table S2: Information on ten selected hub unigenes and endogenous control gene used for RTqPCR analysis. 
392 Table S3: Statistics of sequencing data and assembly results before filtering.

393 Table S4: Function annotation of unigenes based on GO and KEGG databases.

394 Table S5: RT-qPCR verification of the transcriptome results.

395 Fig S1: Sampling positions on the cuttings of $P$. yunnanensis.

396 Fig S2: BLAST results of unigenes in the NR database. (A) E-value distribution statistics. (B) 397 Similarity distribution statistics. (C) Species distribution statistics.

398 Fig S3: RT-qPCR verification of ten selected hub unigenes in comparison with the transcriptome 399 results. The normalized RT-qPCR data are given as the means \pm standard errors (SEs) of three 400 biological replicates.

$401 *$ *zip file 1: Cytoscape working files.zip.

402 *.zip file 2: DEGs detection.zip.

403 Raw data: The sequencing data are available in the link

404 "https://figshare.com/s/8f2fb759e2ee5e5d0778" and the DOI "10.6084/m9.figshare.7973858".

405

406

407

408

409

410

411

412

413

414

415

416

417

418

419

420

421

422

423

424

425

426

427

428

429

430

431

\section{References}

Ahanger MA, Ashraf M, Bajguz A, Ahmad P. 2018. Brassinosteroids regulate growth in plants under stressful environments and crosstalk with other potential phytohormones. Journal of Plant Growth Regulation 37(4): 1007-1024 DOI: 10.1007/s00344-018-9855-2.

Altschul SF, Gish W, Miller W, Myers EW, Lipman DJ. 1990. Basic local alignment search tool. Journal of Molecular Biology 215(3): 403-410 DOI: 10.1016/S0022-2836(05)80360-2.

Anders S, Huber W. 2010. Differential expression analysis for sequence count data. Genome Biology 11(10): R106 DOI: 10.1186/gb-2010-11-10-r106.

Bakshi A, Shemansky JM, Chang C, Binder BM. 2015. History of research on the plant hormone ethylene. Journal of Plant Growth Regulation 34(4): 809-827 DOI: 10.1007/s00344-0159522-9.

Band LR, Ubeda-Tomas S, Dyson RJ, Middleton AM, Hodgman TC, Owen MR, Jensen OE, Bennett MJ, King JR. 2012. Growth-induced hormone dilution can explain the dynamics of plant root cell elongation. Proceedings of the National Academy of Sciences of the United States of America 109(19): 7577-7582 DOI: 10.1073/pnas.1113632109.

Baskin TI, Peret B, Baluška F, Benfey PN, Bennett M, Forde BG, Gilroy S, Helariutta Y, Hepler PK, Leyser O, Masson PH, Muday GK, Murphy AS, Poethig S, Rahman A, Roberts K, Scheres B, Sharp RE, Somerville C. 2010. Shootward and rootward: peak terminology for plant polarity. Trends in Plant Science 15(11): 593-594 DOI: 10.1016/j.tplants.2010.08.006. Belanger KD, Quatrano RS. 2000. Polarity: the role of localized secretion. Current Opinion in Plant Biology 3(1): 67-72 DOI: 10.1016/S1369-5266(99)00043-6.

Belkhadir Y, Jaillais Y. 2015. The molecular circuitry of brassinosteroid signaling. New Phytologist 206(2): 522-540 DOI: 10.1111/nph.13269.

Berrabah F, Balliau T, Ait-Salem EIH, George J, Zivy M, Ratet P, Gourion B. 2018. Control of the ethylene signaling pathway prevents plant defenses during intracellular accommodation of the rhizobia. New Phytologist 219(1): 310-323 DOI: 10.1111/nph.15142. 
432 Bishop GJ. 2003. Brassinosteroid mutants of crops. Journal of Plant Growth Regulation 22(4): 433 325-335 DOI: 10.1007/s00344-003-0064-1.

434 Bloch R. 1943. Polarity in plants. The Botanical Review 9: 261-310 DOI:

$435 \quad 10.1007 \% 2$ FBF02872477.

436

437

438

439

440

441

442

443

444

445

446

447

448

449

450

451

452

453

454

455

456

457

458

459

460

461

462

463

464

465

466

467

468

469

470

Bornens M. 2018. Cell polarity: having and making sense of direction - on the evolutionary significance of the primary cilium/centrosome organ in Metazoa. Open Biology 8(8): 180052 DOI: $10.1098 /$ rsob.180052.

Bringmann M, Bergmann DC. 2017. Tissue-wide mechanical forces influence the polarity of stomatal stem cells in Arabidopsis. Current Biology 27(6): 877-883 DOI: 10.1016/j.cub.2017.01.059.

Broekgaarden C, Caarls L, Vos IA, Pieterse CMJ, Van Wees SCM. 2015. Ethylene: traffic controller on hormonal crossroads to defense. Plant Physiology 169: 2371-2397 DOI: 10.1104/pp.15.01020.

Brownlee C, Bouget FY, Corellou F. 2001. Choosing sides: establishment of polarity in zygotes of fucoid algae. Seminars in Cell \& Developmental Biology 12(5): 345-351 DOI: $10.1006 / \mathrm{scdb} .2001 .0262$.

Bünning E. 1952. Morphogenesis in plants. Survey of Biological Progress 2: 105-140 DOI: 10.1016/B978-1-4832-0001-9.50007-5

Caño-Delgado A, Yin YH, Yu C, Vafeados D, Mora-García S, Cheng JC, Nam KH, Li JM, Chory J. 2004. BRL1 and BRL3 are novel brassinosteroid receptors that function in vascular differentiation in Arabidopsis. Development 131(21): 5341-5351 DOI: 10.1242/dev.01403.

Carraro N, Tisdale-Orr TE, Clouse RM, Knöller AS, Spicer R. 2012. Diversification and expression of the PIN, AUX/LAX, and ABCB Families of putative auxin transporters in Populus. Frontiers in Plant Science 3: 17 DOI: 10.3389/fpls.2012.00017.

Chang C. 2003. Ethylene signaling: the MAPK module has finally landed. Trends in Plant Science 8(8): 365-368 DOI: 10.1016/S1360-1385(03)00156-0.

Chaparro-Garcia A, Wilkinson RC, Gimenez-Ibanez S, Findlay K, Coffey MD, Zipfel C, Rathjen JP, Kamoun S, Schornack S. 2011. The receptor-like kinase SERK3/BAK1 is required for basal resistance against the late blight pathogen Phytophthora infestans in Nicotiana benthamiana. PLoS One 6(1): e16608 DOI: 10.1371/journal.pone.0016608.

Chen CJ, Xia R, Chen H, He YH. 2018. TBtools, a Toolkit for Biologists integrating various HTS - data handling tools with a user-friendly interface. BioRxiv. DOI: 10.1101/289660.

Chen LH, Zhang S, Zhao HX, Korpelainen H, Li CY. 2010. Sex-related adaptive responses to interaction of drought and salinity in Populus yunnanensis. Plant Cell and Environment 33(10): 1767-1778 DOI: 10.1111/j.1365-3040.2010.02182.x.

Chinchilla D, Zipfel C, Robatzek S, Kemmerling B, Nurnberger T, Jones JDG, Felix G, Boller T. 2007. A flagellin-induced complex of the receptor FLS2 and BAK1 initiates plant defence. Nature 448(7152): 497-501 DOI: 10.1038/nature05999.

Peer] reviewing PDF | (2019:04:36443:2:1:NEW 9 Aug 2019) 
471 Cline MS, Smoot M, Cerami E, Kuchinsky A, Landys N, Workman C, Christmas R, Avila472 Campilo I, Creech M, Gross B, Hanspers K, Isserlin R, Kelley R, Killcoyne S, Lotia S,

473

474

475

476

477

478

479

480

481

482

483

484

485

486

487

488

489

490

491

492

493

494

495

496

497

498

499

500

501

502

503

504

505

506

507

508

509

510 Maere S, Morris J, Ono K, Pavlovic V, Rico AR, Vailaya A, Wang PL, Adler A, Conklin BR, Hood L, Kuiper M, Sander C, Schmulevich I, Schwikowski B, Warner GJ, Ideker T, Bader GD. 2007. Integration of biological networks and gene expression data using Cytoscape. Nature Protocols 2(10): 2366-2382 DOI: 10.1038/nprot.2007.324.

Clouse SD, Langford M, McMorris TC. 1996. A brassinosteroid-insensitive mutant in Arabidopsis thaliana exhibits multiple defects in growth and development. Plant Physiology 111(3): 671-678 DOI: 10.1104/pp.111.3.671.

Clouse SD, Sasse JM. 1998. Brassinosteroids: essential regulators of plant growth and development. Annual Review of Plant Physiology and Plant Molecular Biology 49(1): 427451 DOI: 10.1146/annurev.arplant.49.1.427.

Colcombet J, Hirt H. 2008. Arabidopsis MAPKs: a complex signalling network involved in multiple biological processes. Biochemical Journal 413(2): 217-226 DOI: 10.1042/BJ20080625.

Corbineau F, Xia Q, Bailly C, EI-Maarouf-Bouteau H. 2014. Ethylene, a key factor in the regulation of seed dormancy. Front in Plant Science 5: 539 DOI: 10.3389/fpls.2014.00539.

De Bruyne L, Höfte M, De Vleesschauwer D. 2014. Connecting growth and defense: the emerging roles of brassinosteroids and gibberellins in plant innate immunity. Molecular Plant 7(6): $943-959$ DOI: $10.1093 / \mathrm{mp} / \mathrm{ssu} 050$.

Depuydt S, Hardtke CS. 2011. Hormone signaling crosstalk in plant growth regulation. Current Biology 21(9): 365-373 DOI: 10.1016/j.cub.2011.03.013.

Euchen EE, Fox S, de Reuille PB, Kennaway R, Bensmihen S, Avondo J, Calder GM, Southam P, Robinson S, Bangham A, Coen E. 2012. Generation of leaf shape through early patterns of growth and tissue polarity. Science 335(6072): 1092-1096 DOI:

10.1126/science. 1214678.

Friml J, Benfey P, Benková E, Bennett M, Berleth T, Geldner N, Grebe M, Heisler M, Hejátko J, Jürgens G, Laux T, Lindsey K, Lukowitz W, Luschnig C, Offringa R, Scheres B, Swarup R, Torres-Ruiz R, Weijers D, Zažímalová E. 2006. Apical-basal polarity: why plant cells don’t stand on their heads. Trends in Plant Science 11(1): 12-14 DOI:

10.1016/j.tplants.2005.11.010.

Fukuda H. 2004. Signals that control plant vascular cell differentiation. Nature Reviews Molecular Cell Biology 5(5): 379-391 DOI: 10.1038/nrm1364.

Grabherr MG, Grabherr MG, Haas BJ, Yassour M, Levin JZ, Thompson DA, Amit I, Adiconis X, Fan L, Raychowdhury R, Zeng QD, Chen ZH, Mauceli E, Hacohen N, Gnirke A, Bhind N, di Palma F, Birren BW, Nusbaum C, Lindblad-Toh K, Friedman N, Regev A. 2011. Full-length transcriptome assembly from RNA-Seq data without a reference genome. Nature Biotechnology 29(7): 644-652 DOI: 10.1038/nbt. 1883.

Grebe M. 2004. Ups and downs of tissue and planar polarity in plants. BioEssays 26(7): 719-729 DOI: 10.1002/bies.20065. 
511 Hable WE, Hart PE. 2010. Signaling mechanisms in the establishment of plant and fucoid algal

512

513

514

515

516

517

518

519

520

521

522

523

524

525

526

527

528

529

530

531

532

533

534

535

536

537

538

539

540

541

542

543

544

545

546

547

548

549

550 10.1002/mrd.21199.

Heese A, Hann DR, Gimenez-Ibanez S, Jones AME, He K, Li J, Schroeder JI, Peck SC, Rathjen J. 2007. The receptor-like kinase SERK3/BAK1 is a central regulator of innate immunity in plants. Proceedings of the National Academy of Sciences of the United States of America 104(29): 12217-12222 DOI: 10.1073pnas.0705306104.

Henry E, Yadeta KA, Coaker G. 2013. Recognition of bacterial plant pathogens: local, systemic and transgenerational immunity. New Phytologist 199(4): 908-915 DOI: 10.1111/nph.12214.

Hua J, Meyorowitz EM. 1998. Ethylene responses are negatively regulated by a receptor gene family in Arabidopsis thaliana. Cell 94(2): 261-271 DOI: 10.1016/S0092-8674(00)81425-7.

Jiang H, Korpelainen H, Li CY. 2013. Populus yunnanensis males adopt more efficient protective strategies than females to cope with excess zinc and acid rain. Chemosphere 91(8): 1213-1220 DOI: 10.1016/j.chemosphere.2013.01.041.

Jiang H, Peng SM, Zhang S, Li XG, Korpelainen H, Li CY. 2012. Transcriptional profiling analysis in Populus yunnanensis provides insights into molecular mechanisms of sexual differences in salinity tolerance. Journal of Experimental Botany 63(10): 3709-3726 DOI: 10.1093/jxb/ers064.

Krishna P. 2003. Brassinosteroid-mediated stress responses. Journal of Plant Growth Regulation 22(4): 289-297 DOI: 10.1007/s00344-003-0058-z.

Lalitha S. 2000. Primer Premier 5. Biotech Software \& Internet Report 1(6): 270-272 DOI: $10.1089 / 152791600459894$.

Langfelder P, Horvath S. 2008. WGCNA: an R package for weighted correlation network analysis. BMC Bioinformatics 9: 559 DOI: 10.1186/1471-2105-9-559.

Li B, Dewey CN. 2011. RSEM: Accurate transcript quantification from RNA-Seq data with or without a reference genome. BMC Bioinformatics 12: 323 DOI: 10.1186/1471-2105-12-323.

Li J, Wen JQ, Lease KA, Doke JT, Tax FE, Walker JC. 2002. BAK1, an Arabidopsis LRR receptor-like protein kinase, interacts with BRI1 and modulates brassinosteroid signaling. Cell 110(2): 213-222 DOI: 10.1016/S0092-8674(02)00812-7.

Li S, Han XF, Yang LY, Deng XX, Wu HJ, Zhang MM, Liu YD, Zhang SQ, Xu J. 2018. Mitogen-activated protein kinases and calcium-dependent protein kinases are involved in wounding-induced ethylene biosynthesis in Arabidopsis thaliana. Plant Cell and Environment 41(1): 134-147 DOI: 10.1111/pce.12984.

Li X, Yang YQ, Sun XD, Lin HM, Chen JH, Ren J, Hu XY, Yang YP. 2014. Comparative physiological and proteomic analyses of poplar (Populus yunnanensis) plantlets exposed to high temperature and drought. PLoS One 9(9): e107605 DOI: 10.1371/journal.pone.0107605.

Livak KJ, Schmittgen TD. 2001. Analysis of relative gene expression data using realtime quantitative PCR and the $2^{-\Delta \Delta \mathrm{Ct}}$ method. Methods 25(4): 402-408 DOI: 10.1006/meth.2001.1262. 
551

552

553

554

555

556

557

558

559

560

561

562

563

564

565

566

567

568

569

570

571

572

573

574

575

576

577

578

579

580

581

582

583

584

585

586

587

588

589

590

Lozano-Duran R, Zipfel C. 2015. Trade-off between growth and immunity: role of brassinosteroids. Trends in Plant Science 20(1): 12-19 DOI: 10.1016/j.tplants.2014.09.003.

Mandava NB. 1988. Plant growth-promoting brassinosteroids. Annual Review of Plant Physiology and Plant Molecular Biology 39(1): 23-52 DOI:

10.1146/annurev.pp.39.060188.000323.

Medvedev SS. 2012. Mechanisms and physiological role of polarity in plants. Russian Journal of Plant Physiology 59(4): 502-514 DOI: 10.1134/S1021443712040085.

Meng XZ, Zhang SQ. 2013. MAPK cascades in plant disease resistance signaling. Annual Review of Phytopathology 51(1): 245-266 DOI: 10.1146/annurev-phyto-082712-102314.

Muday GK, Rahman A, Binder BM. 2012. Auxin and ethylene: collaborators or competitors? Trends in Plant Science 17(4): 181-195 DOI: 10.1016/j.tplants.2012.02.001.

Müller M, Munné-Bosch S. 2015. Ethylene response factors: a key regulatory hub in hormone and stress signaling. Plant Physiology 169(1): 32-41 DOI: 10.1104/pp.15.00677

Nam KH, Li JM. 2002. BRI1/BAK1, a receptor kinase pair mediating brassinosteroid signaling. Cell 110(2): 203-212 DOI: 10.1016/S0092-8674(02)00814-0.

Nick P, Furuya M. 1992. Induction and fixation of polarity - early steps in plant morphogenesis. Development, Growth and Differentiation 34(2): 115-125 DOI: 10.1111/j.1440169X.1992.tb00001.x.

Ouaked F, Wilfried R, Lecourieux D, Hirt H. 2003. A MAPK pathway mediates ethylene signaling in plants. The EMBO Journal 22(6): 1282-1288 DOI: 10.1093/emboj/cdg131.

Pacifici E, Polverari L, Sabatini S. 2015. Plant hormone cross-talk: the pivot of root growth. Journal of Experimental Botany 66(4): 1113-1121 DOI: 10.1093/jxb/eru534.

Pertea G, Huang X, Liang F, Antonescu V, Sultana R, Karamycheva S, Lee Y, White J, Cheung F, Parvizi B, Tsai J, Quackenbush J. 2003. TIGR Gene Indices clustering tools (TGICL): a software system for fast clustering of large EST datasets. Bioinformatics 19(5): 651-652 DOI: 10.1093/bioinformatics/btg034.

Qi JY, Wu BB, Feng SL, Lv SQ, Guan CM, Zhang X, Qiu DL, Hu YC, Zhou YH, Li CY, Long M, Jiao YL. 2017. Mechanical regulation of organ asymmetry in leaves. Nature plants 3(9): 724-733 DOI: 10.1038/s41477-017-0008-6.

Qu X, Hall B, Gao Z, Schaller GE. 2007. A strong constitutive ethylene-response phenotype conferred on Arabidopsis plants containing null mutations in the ethylene receptors ETR1 and ERS1. BMC Plant Biology 7(1): 3 DOI: 10.1186/1471-2229-7-3.

Ren J, Dai WR, Yang CM, Ma XL, Zou CB. 2018. Physiological regulation of poplar species to experimental warming differs between species with contrasting elevation ranges. New Forests 49(3): 329-340 DOI: 10.1007/s11056-017-9622-4.

Sachs T. 1969. Polarity and the induction of organized vascular tissues. Annals of Botany 33(2): 263-275 DOI: 10.1093/oxfordjournals.aob.a084281.

Santner A, Calderon-Villalobos LI, Estelle M. 2009. Plant hormones are versatile chemical regulators of plant growth. Nature Chemical Biology 5(5): 301-307 DOI: 10.1038/nchembio. 165 . 
591 Santner A, Estelle M. 2009. Recent advances and emerging trends in plant hormone signaling.

592

593

594

595

596

597

598

599

600

601

602

603

604

605

606

607

608

609

610

611

612

613

614

615

616

617

618

619

620

621

622

623

624

625

626

627

628

629 Nature 459(7250): 1071-1078 DOI: 10.1038/nature08122.

Schweighofer A, Meskiene I. 2008. Regulation of stress hormones jasmonates and ethylene by MAPK pathways in plants. Molecular BioSystems 4(8): 799-803 DOI: 10.1039/b718578m. Shannon P, Markiel A, Ozier O, Baliga NS, Wang JT, Ramage D, Amin N, Schwikowski B, Ideker T. 2003. Cytoscape: A software environment for integrated models of biomolecular interaction networks. Genome Research 13(11): 2498-2504 DOI: 10.1101/gr.1239303.

Singh AP, Savaldi-Goldstein S. 2015. Growth control: brassinosteroid activity gets context. Journal of Experimental Botany 66(4): 1123-1132 DOI: 10.1093/jxb/erv026.

Souter M, Lindsey K. 2000. Polarity and signaling in plant embryogenesis. Journal of Experimental Botany 51(347): 971-983 DOI: 10.1093/jexbot/51.347.971.

Strzyz P. 2017. Forcing cell polarity. Nature Reviews Molecular Cell Biology 18(5): 278-278 DOI: $10.1038 / \mathrm{nrm} .2017 .34$.

Thao NP, Khan MIR, Thu NBA, Hoang XLT, Asgher M, Khan NA, Tran LP. 2015. Role of ethylene and its cross talk with other signaling molecules in plant responses to heavy metal stress. Plant Physiology 169(1): 73-84 DOI: 10.1104/pp.15.00663.

Tian HY, Lv BS, Ding TT, Bai MY, Ding ZJ. 2018. Auxin-BR interaction regulates plant growth and development. Front in Plant Science 8: 2256 DOI: 10.3389/fpls.2017.02256.

Vragovic K, Sela A, Friedlander-Shani L, Fridman Y, Hacham Y, Holland N, Bartom E, Mockler TC, Savaldi-Goldstein S. 2015. Translatome analyses capture of opposing tissuespecific brassinosteroid signals orchestrating root meristem differentiation. Proceedings of the National Academy of Sciences of the United States of America 112(3): 923-928 DOI: 10.1073/pnas. 1417947112 .

Weisenseel MH. 1979. Induction of polarity. In: Haupt W, Fenleib ME, eds. Encyclopedia of plant physiology. Berlin: Springer, 485-505.

Went FW. 1941. Polarity of auxin transport in inverted Tagetes cuttings. Botanical Gazette 103(2): 386-390 DOI: 10.1086/335050.

Wickham H. 2016. ggplot2 - elegant graphics of data analysis. Berlin: Springer.

Yamada K, Yamashita-Yamada M, Hirase T, Fujiwara T, Tsuda K, Hiruma K, Saijo Y. 2016. Danger peptide receptor signaling in plants ensures basal immunity upon pathogen-induced depletion of BAK1. The EMBO Journal 35(1): 46-61 DOI: 10.15252/embj.201591807.

Yasuda S, Okada K, Saijo Y. 2017. A look at plant immunity through the window of the multitasking coreceptor BAK1. Current Opinion in Plant Biology 38: 10-18 DOI: 10.1016/j.pbi.2017.04.007.

Yun T, Li JM, Xu Y, Zhou AP, Wang S, Li D, He CZ. 2019. Selection of reference genes for RT-qPCR analysis in the bark of Populus yunnanensis cuttings. Journal of Environmental Biology 40(3): 584-591 DOI: 10.22438/jeb/40/3(SI)/Sp-24.

Zhou AP, Zong D, Gan PH, Zou XL, Fei X, Zhong YY, He CZ. 2018. Physiological analysis and transcriptome profiling of inverted cuttings of Populus yunnanensis reveal that cell wall 
630 metabolism plays a crucial role in responding to inversion. Genes 9(12): 572 DOI:

$631 \quad 10.3390 /$ genes9120572.

632 
633 Table 1 Information on the top 20 hub unigenes. "NA" indicates that the unigenes were not 634 involved in the plant hormone signal transduction pathway.

635 Fig. 1 Vegetative form of $P$. yunnanensis cuttings. (A) Upright cuttings. (B) Inverted cuttings.

636 Fig. 2 Functional annotations of unigenes before and after filtering.

637 Fig. 3 DEGs between upright and inverted cuttings. BU indicates position B of upright cuttings. $638 \mathrm{CU}$ indicates position $\mathrm{C}$ of upright cuttings. DU indicates position D of upright cuttings. EU 639 indicates position $\mathrm{E}$ of upright cuttings. BI indicates position B of inverted cuttings. CI indicates 640 position $\mathrm{C}$ of inverted cuttings. DI indicates position D of inverted cuttings. EI indicates position 641 E of inverted cuttings.

642 Fig. 4 GO classification and term enrichment of DEGs. (A) BU vs BI. (B) BU vs CI. (C) CU vs 643 CI. (D) CU vs BI.

644 Fig. 5 KEGG pathway enrichment of DEGs. (A) BU vs BI. (B) BU vs CI. (C) CU vs CI. (D) CU 645 vs BI.

646 Fig. 6 DEG information in four pairs. (A, B, C, D) BU vs BI. (E, F, G, H) BU vs CI. (I, J, K, L) 647 CU vs CI. (M, N, O, P) CU vs BI. (A, E, I, M) Venn diagram of DEGs in four pathways: plant 648 hormone signal transduction, MAPK signaling pathway-plant, plant-pathogen interaction and 649 ABC transporters. (B, F, J, N) Number of unigenes assigned to orthology in hormone signaling. 650 (C, G, K, O) Boxplot of expression levels of DEGs involved in hormone signaling. (D, H, L, P) 651 Expression heatmap of DEGs involved in hormone signaling.

652 Fig. 7 WGCNA of four KEGG pathways. (A, B) Hierarchical clustering dendrogram of samples. 653 (C, D) Scatter determining the soft threshold. (E) Hierarchical clustering of unigenes and module 654 identification. (F) Network heatmap plot based on 500 randomly selected genes. The 655 progressively more saturated yellow and red colors indicate high coexpression 656 interconnectedness. (G) Relationships between gene modules and sample groups. (H) 657 Hierarchical clustering of gene modules and sample groups. (I) Correlation heatmap of gene 658 modules and sample groups.

659 Fig. 8 Network of unigenes in modules highly connected to sample groups. (A) Network of 660 unigenes in the turquoise module, which is highly connected to the BU group. The unigenes 661 aligned to the plant hormone signal transduction pathway are amplified to show detail. (B) 662 Network of unigenes in the lightgreen module, which is highly connected to the BI group. (C) 663 Network of unigenes in the darkturquoise and orangered4 modules, which are highly connected 664 to the $\mathrm{CU}$ group. (D) Network of unigenes in the paleturquoise module, which is highly 665 connected to the CI group. Each node indicates a gene, and its size indicates the number of hits 666 to this gene. The $\log _{2} \mathrm{FC}$ values of the unigenes are characterized by five colors: green, showing $667 \log _{2} \mathrm{FC}<-2$; blue, showing $-2 \leq \log _{2} \mathrm{FC}<-1$; pink, showing $-1 \leq \log _{2} \mathrm{FC} \leq 1$; orange, showing 1

$668<\log _{2} \mathrm{FC} \leq 2$; and red, showing $2<\log _{2} \mathrm{FC}$. The unigene names are provided when $\mathrm{Q}$ values $<$ 669 0.05, and two sizes are used based on different significance levels: the larger indicates a 670 significant expression change at the 0.01 level, and the smaller size indicates a significant 671 expression change at the 0.05 level.

672 


\section{Table $\mathbf{1}$ (on next page)}

Information on the top 20 hub unigenes.

"NA" indicates that the unigenes were not involved in the plant hormone signal transduction pathway. 
1 Table 1 Information on the top 20 hub unigenes. "NA" indicates that the unigenes were involved to the plant 2 hormone signal transduction pathway.

\begin{tabular}{llllr}
\hline Gene module & Plant hormone signal transduction & KEGG & KO ID & $\begin{array}{l}\text { Number of } \\
\text { unigenes }\end{array}$ \\
\hline pathway & Orthology & & 8 \\
& Brassinosteroid & BAK1 & K13416 & 4 \\
& Abscisic acid & PP2C & K14497 & 4 \\
& Jasmonic acid & MYC2 & K13422 & 2 \\
& Ethylene & CTR1 & K14510 & 1 \\
& Salicylic acid & SIMKK & K13413 & 1 \\
& Ethylene & PR-1 & K134449 & 8 \\
& & ETR & K14509 & 4 \\
& & EIN3 & K14514 & 3 \\
& & EBF1/2 & K14515 & 1 \\
& Abscisic acid & CTR1 & K14510 & 1 \\
& NA & PP2C & K14497 & 3 \\
\hline
\end{tabular}

3 
Figure 1

Vegetative form of $P$. yunnanensis cuttings.

(A) Upright cuttings. (B) Inverted cuttings. 


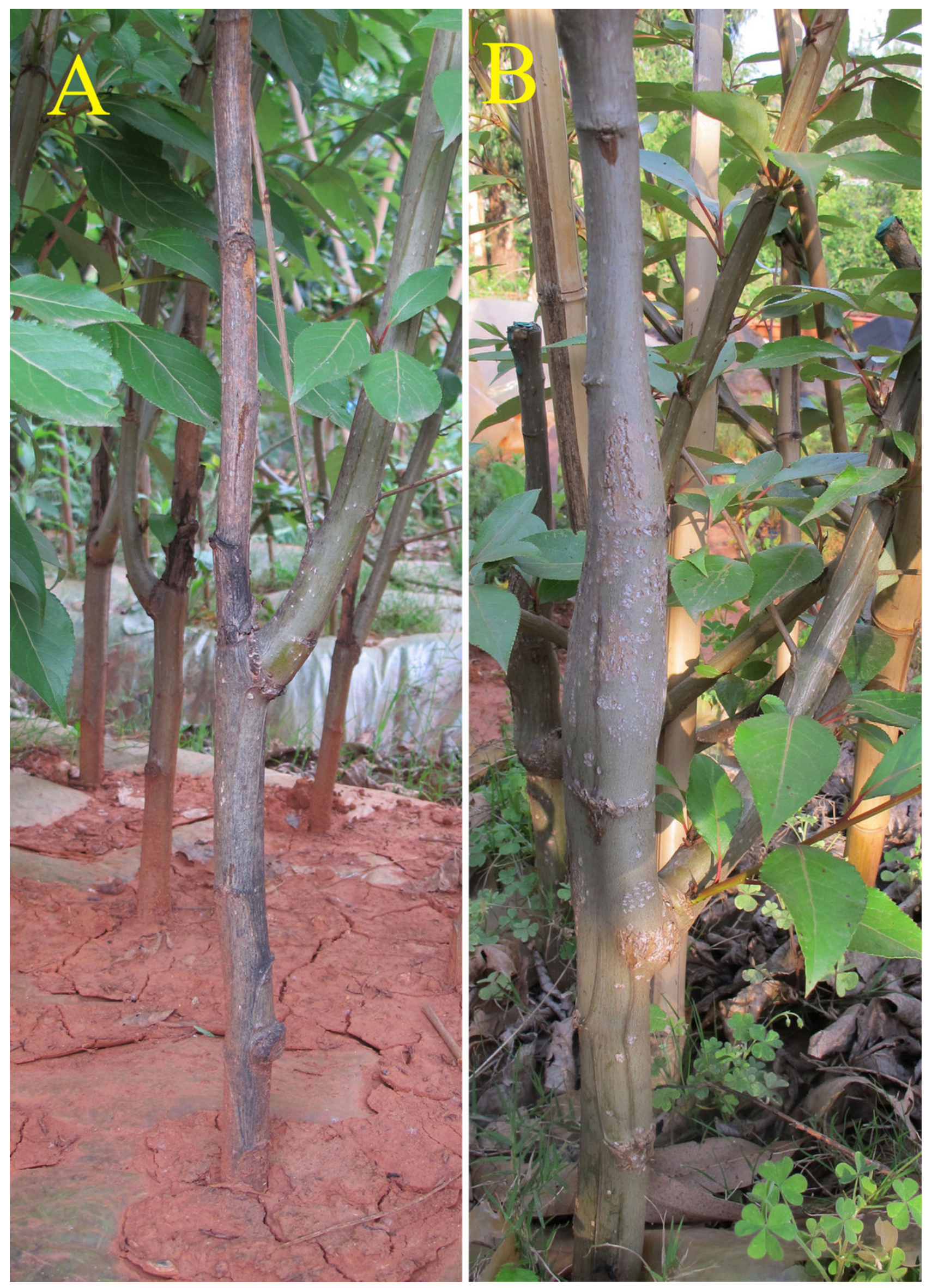


Figure 2

Functional annotations of unigenes before and after filtering.

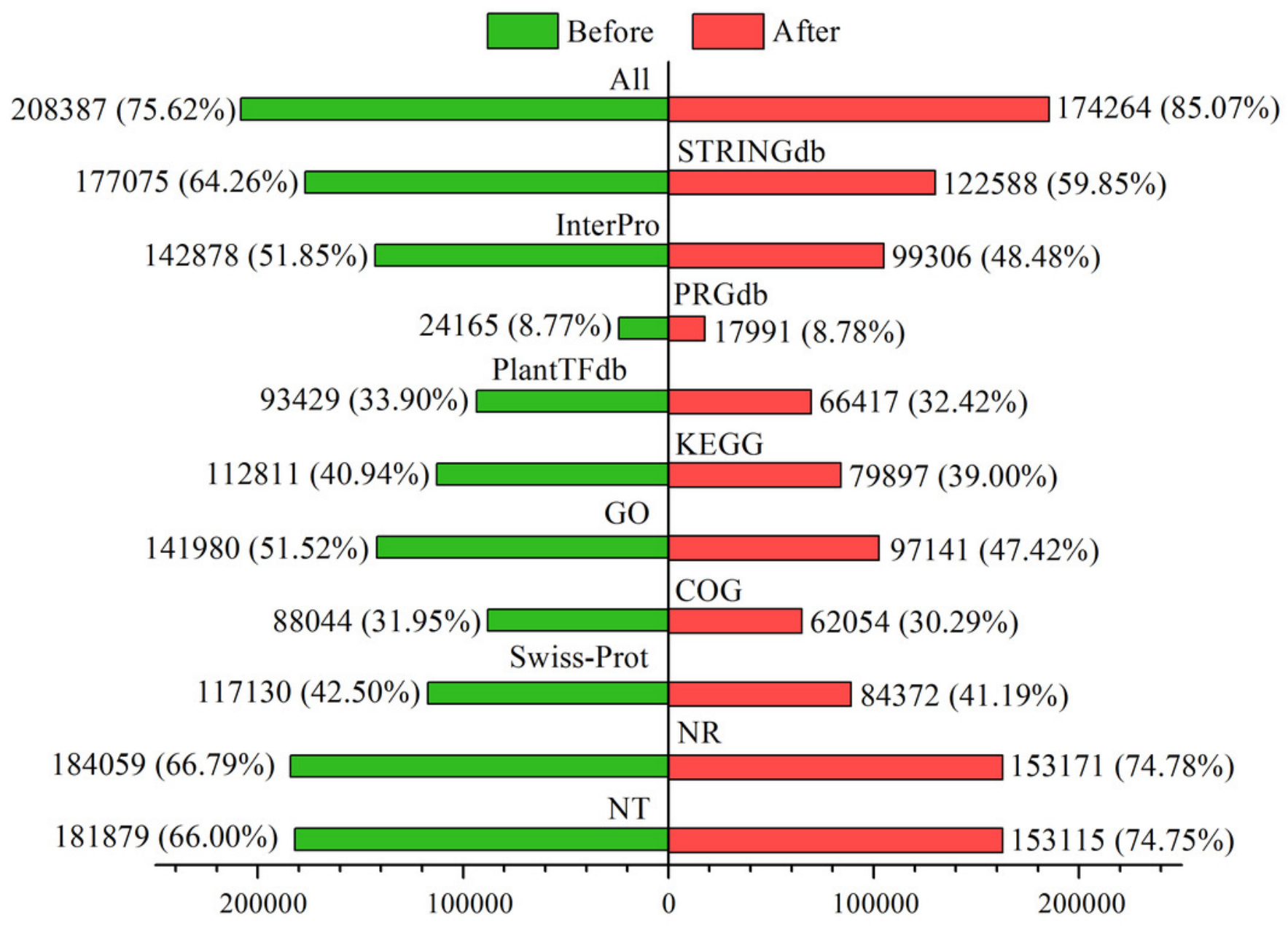




\section{Figure 3}

DEGs between upright and inverted cuttings.

BU indicates position B of upright cuttings. CU indicates position C of upright cuttings. DU indicates position D of upright cuttings. EU indicates position E of upright cuttings. BI indicates position $\mathrm{B}$ of inverted cuttings. $\mathrm{Cl}$ indicates position $\mathrm{C}$ of inverted cuttings. DI indicates position $D$ of inverted cuttings. El indicates position $E$ of inverted cuttings.

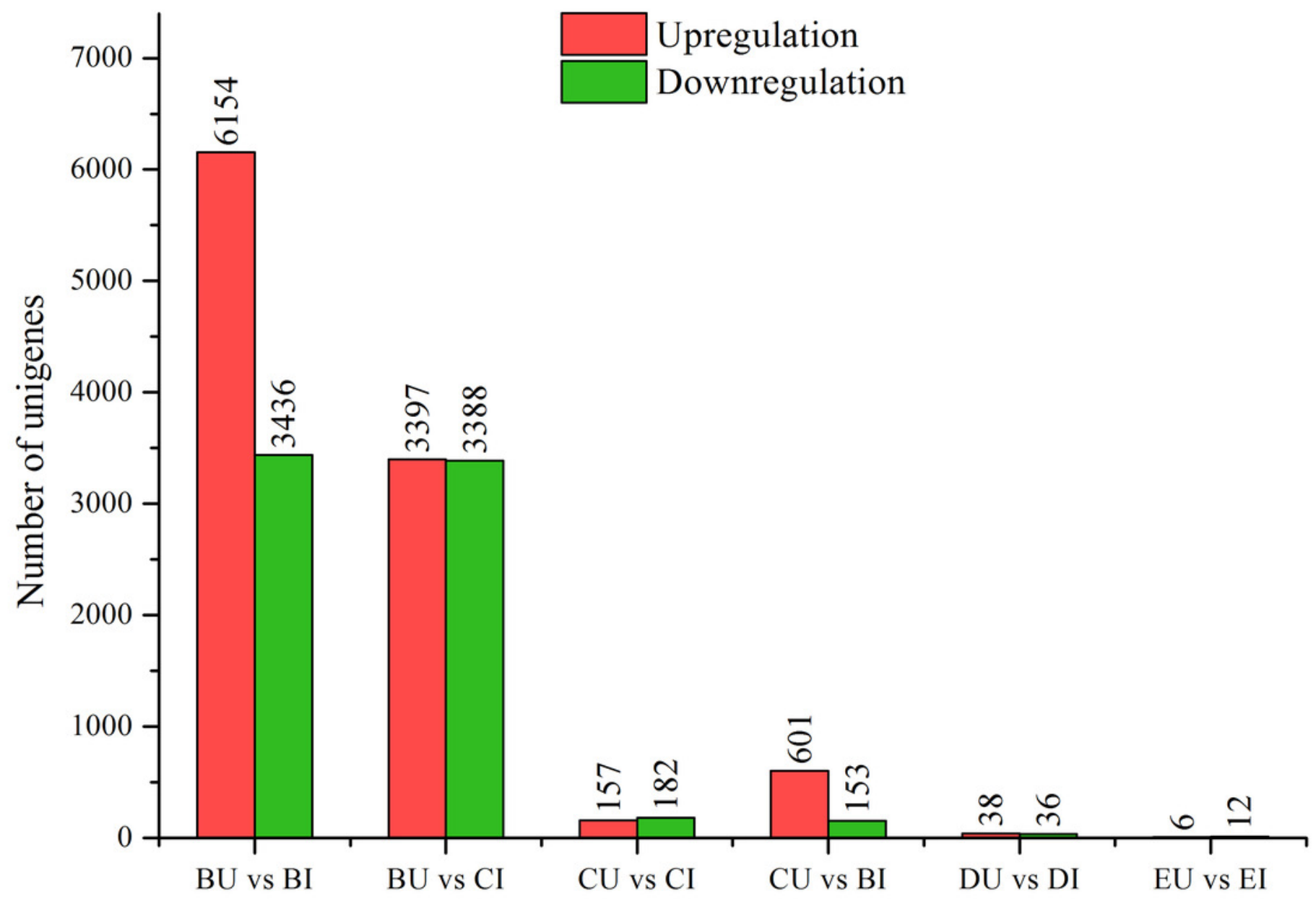


Figure 4

\title{
GO classification and term enrichment of DEGs.
}

\author{
(A) $\mathrm{BU}$ vs $\mathrm{BI}$. (B) BU vs $\mathrm{Cl}$. (C) $\mathrm{CU}$ vs $\mathrm{Cl}$. (D) $\mathrm{CU}$ vs $\mathrm{BI}$.
}

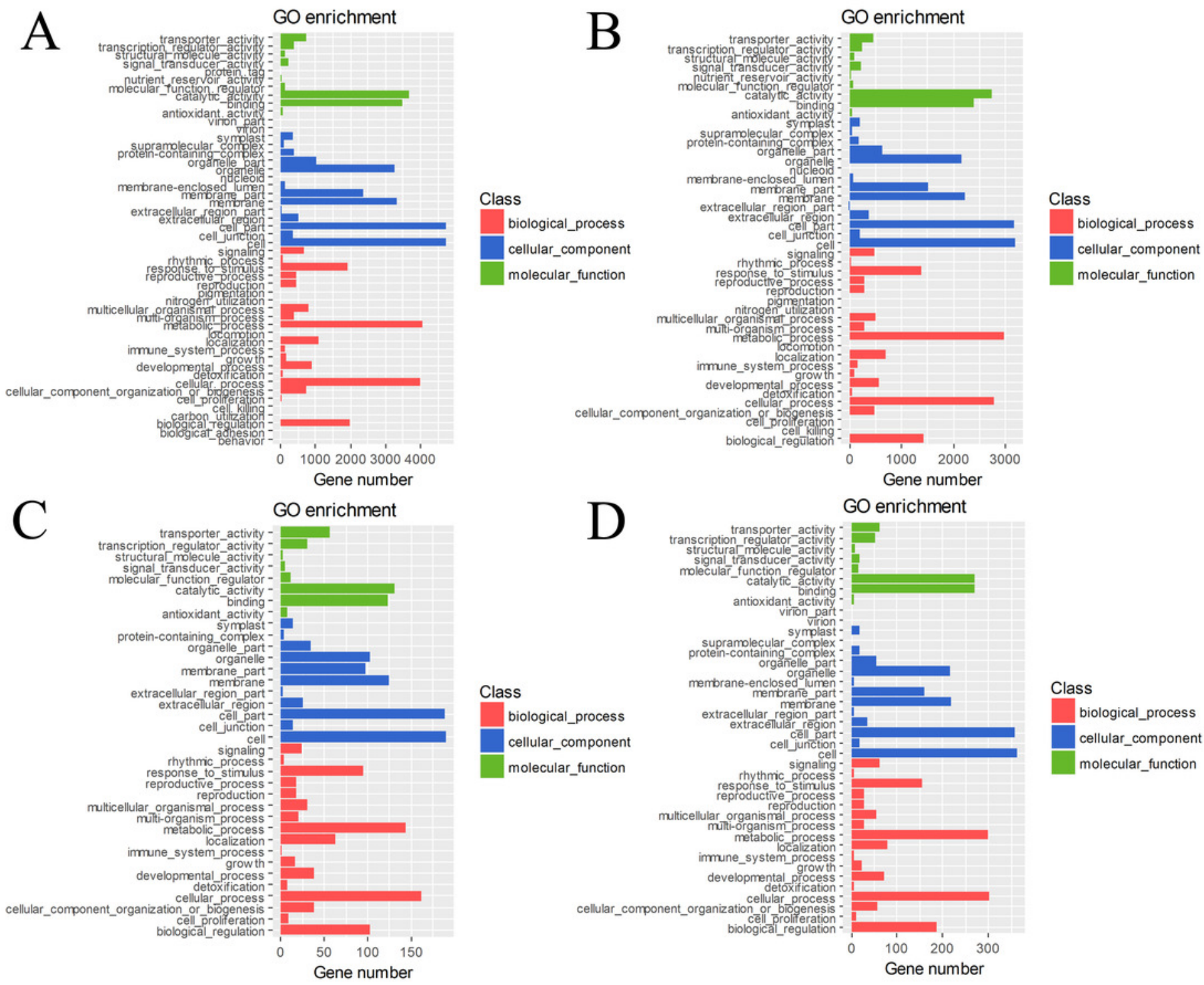


Figure 5

\section{KEGG pathway enrichment of DEGs.}

\section{(A) $\mathrm{BU}$ vs $\mathrm{BI}$. (B) BU vs $\mathrm{Cl}$. (C) $\mathrm{CU}$ vs $\mathrm{Cl}$. (D) $\mathrm{CU}$ vs $\mathrm{BI}$.}

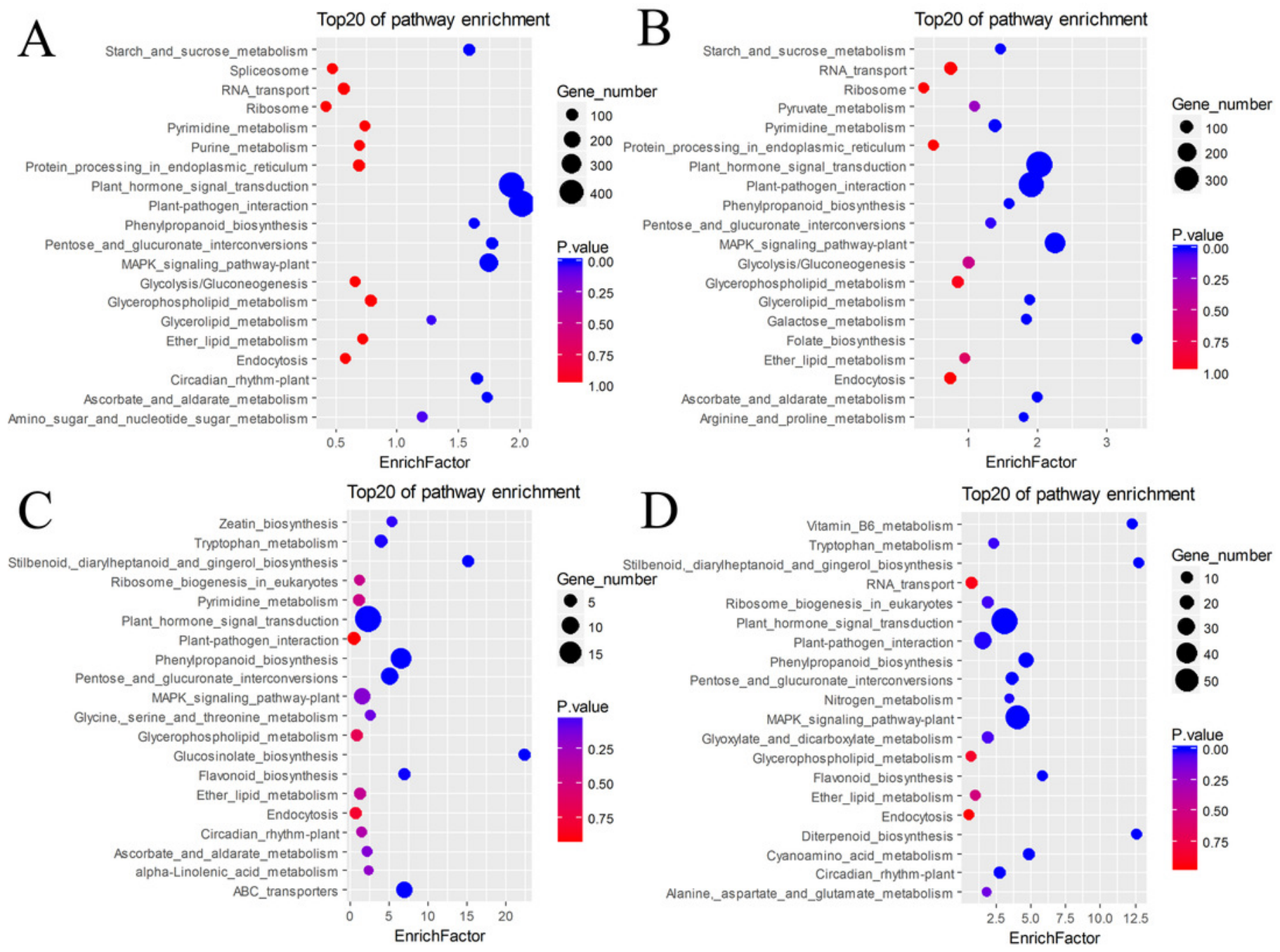




\section{Figure 6}

DEG information in four pairs.

$(A, B, C, D)$ BU vs BI. (E, F, G, H) BU vs $C l$. (I, J, K, L) CU vs Cl. (M, N, O, P) CU vs BI. (A, E, I, M) Venn diagram of DEGs in four pathways: plant hormone signal transduction, MAPK signaling pathway-plant, plant-pathogen interaction and ABC transporters. (B, F, J, N) Number of unigenes assigned to orthology in hormone signaling. $(C, G, K, O)$ Boxplot of expression levels of DEGs involved in hormone signaling. (D, H, L, P) Expression heatmap of DEGs involved in hormone signaling.

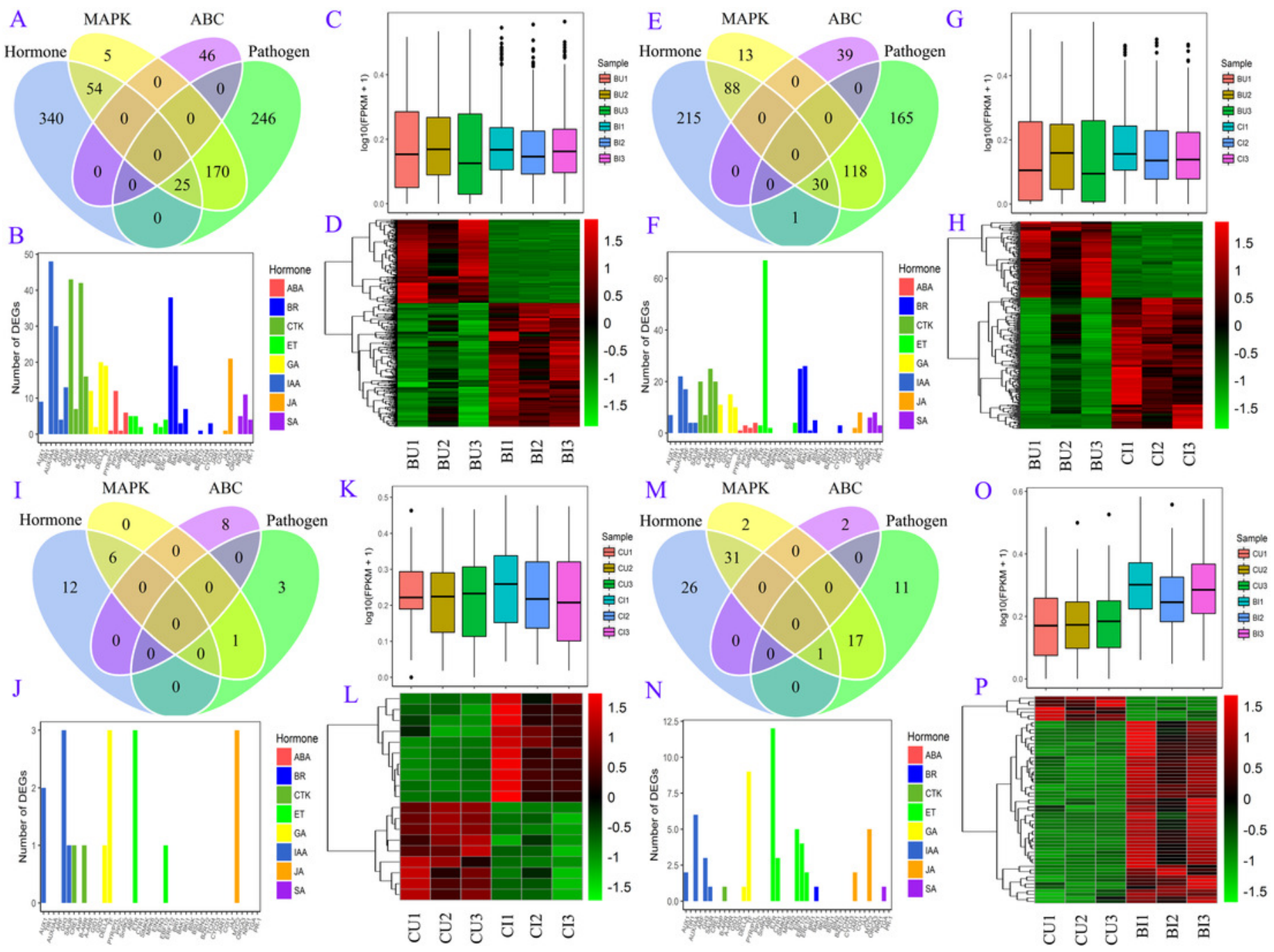




\section{Figure 7}

WGCNA of four KEGG pathways.

(A, B) Hierarchical clustering dendrogram of samples. (C, D) Scatter determining the soft threshold. (E) Hierarchical clustering of unigenes and module identification. (F) Network heatmap plot based on 500 randomly selected genes. The progressively more saturated yellow and red colors indicate high coexpression interconnectedness. (G) Relationships between gene modules and sample groups. (H) Hierarchical clustering of gene modules and sample groups. (I) Correlation heatmap of gene modules and sample groups. 

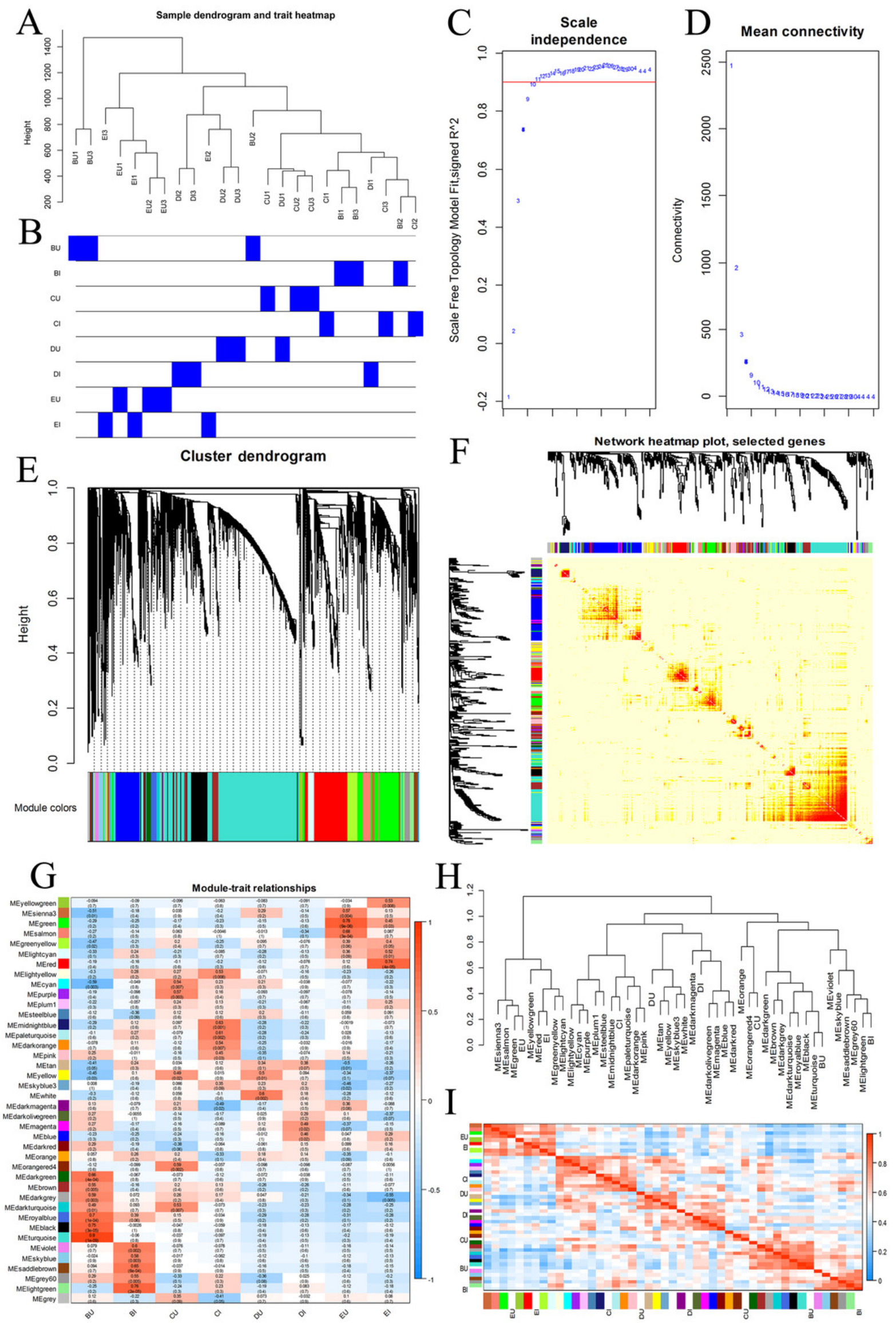


\section{Figure 8}

Network of unigenes in modules highly connected to sample groups.

(A) Network of unigenes in the turquoise module, which is highly connected to the BU group. The unigenes aligned to the plant hormone signal transduction pathway are amplified to show detail. (B) Network of unigenes in the lightgreen module, which is highly connected to the BI group. (C) Network of unigenes in the darkturquoise and orangered4 modules, which are highly connected to the CU group. (D) Network of unigenes in the paleturquoise module, which is highly connected to the $\mathrm{Cl}$ group. Each node indicates a gene, and its size indicates the number of hits to this gene. The $\log _{2} \mathrm{FC}$ values of the unigenes are characterized by five colors: green, showing $\log _{2} \mathrm{FC}<-2$; blue, showing $-2 \leq \log _{2} \mathrm{FC}<-1$; pink, showing $-1 \leq \log _{2} \mathrm{FC}$ $\leq 1$; orange, showing $1<\log _{2} \mathrm{FC} \leq 2$; and red, showing $2<\log _{2} \mathrm{FC}$. The unigene names are provided when $Q$ values $<0.05$, and two sizes are used based on different significance levels: the larger indicates a significant expression change at the 0.01 level, and the smaller size indicates a significant expression change at the 0.05 level. 

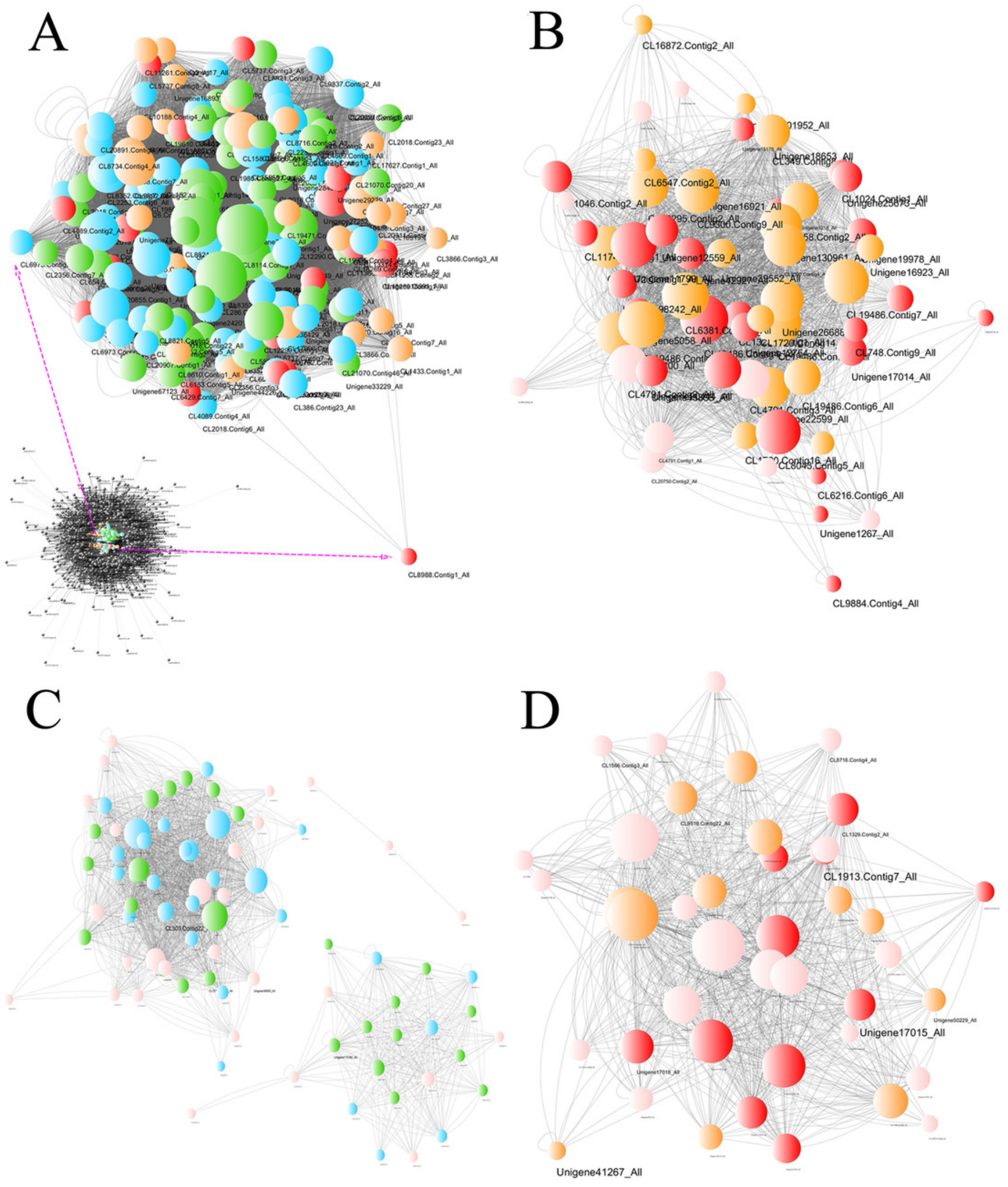